\title{
Torsion of in-utero fetal ovarian cyst
}

\section{Kruthiga Kumari Balasubramanian*, Lalitha Natarajan, Paavai Arunachalam, Latha Maheshwari}

\begin{abstract}
Department of Obstetrics and Gynaecology, PSG Institute of Medical Sciences and Research, Coimbatore, Tamil Nadu, India
\end{abstract}

Received: 17 January 2022

Revised: 09 February 2022

Accepted: 10 February 2022

\section{*Correspondence:}

Dr. Kruthiga Kumari Balasubramanian,

E-mail: sweet6hopes@yahoo.com

Copyright: ( $)$ the author(s), publisher and licensee Medip Academy. This is an open-access article distributed under the terms of the Creative Commons Attribution Non-Commercial License, which permits unrestricted non-commercial use, distribution, and reproduction in any medium, provided the original work is properly cited.

\section{ABSTRACT}

A 22 years old primi-gravida was diagnosed to have a $6.3 \times 5 \times 5.37 \mathrm{~cm}$ hemorrhagic gonadal cyst in fetus at 38 weeks of gestation by growth scan, on follow-up. At term gestation, she delivered a female baby by vacuum assisted vaginal delivery, weighed $2.86 \mathrm{~kg}$, no other anomalies. X-ray done at 1st hour of life showed mass on the right side. USG abdomen done showed an intraperitoneal cystic lesion $(5.7 \times 3.9 \times 6.3 \mathrm{~cm})$ in right lumbar quadrant of abdomen and pediatric surgeon was consulted. Baby taken for diagnostic laparoscopy on second day of life. Findings were right large ovarian cyst with torsion with adhesions to small bowel. $75 \mathrm{ml}$ of hemorrhagic fluid drained and ovarian cystectomy done, sample sent for histopathological examination, turned out to be a simple cyst. Baby discharged on day 7 of life (POD-5), hemodynamically stable and on direct breast feeding, tolerated well.

Keywords: Hemorrhagic gonadal cyst, Torsion, Diagnostic laparoscopy

\section{INTRODUCTION}

Multidisciplinary teamwork is essential to provide the best possible care for patients. This report describes the management of a pregnant woman who was diagnosed with a fetal hemorrhagic abdominal cyst, which can lead to severe complications if not treated timely. This is an interesting, rare case and useful for learning experience.

Ovarian cyst is a fluid filled sac within the ovary, mostly asymptomatic and harmless. The incidence of fetal ovarian cysts detected in utero has increased over the past decade ( 1 in 2600 pregnancies) due to increased use of ultrasound, which is usually diagnosed at the end of second and third trimester scans. Stimulation of fetal ovary by placental and maternal hormones leads to development of ovarian cysts, and usually regress shortly after birth once hormonal stimulation has decreased. ${ }^{1}$
Ovarian cysts can be simple cysts which are completely anechoic in ultrasound, while complex cysts have an echogenic wall, internal septae, debris and blood clots and require surgical management. Complications include torsion, hemorrhage, recurrence, preterm labor and rupture. $^{2}$

From this study, it can be concluded that ultrasound scans done antenatally can help diagnose many anomalies in the fetus in-utero, which help in early interventions postdelivery and prevent further neonatal morbidity and mortality.

\section{CASE REPORT}

22 years old primi gravida, LMP-3/10/20 EDD-10/7/21, non-consanguineous marriage, spontaneous conception, booked and immunized at an outside hospital and undergone regular antenatal checkups, was referred at 38 
weeks of gestation with growth scan findings of an intraabdominal biloculated cyst in the fetus of size $5 \times 5 \mathrm{~cm}$, diagnosed at 35 weeks +5 days of gestation, for further management. Upon arrival in our institution, routine antenatal investigations done. Ultrasound done revealed a multi loculated cyst in the right side of the fetal abdomen of size $6.3 \times 5 \times 5.37 \mathrm{~cm}$, likely a right gonadal cyst with hemorrhage. First and second trimesters- all scans and lab investigations normal. No significant past medical/surgical history, no antenatal risk factors. Pediatric surgeon was consulted on the same day and parents were counselled accordingly.

At 39 weeks +3 days of gestation, she presented with labor pain, spontaneously progressed and delivered a live female baby by vacuum assisted vaginal delivery, weighing 2.86 $\mathrm{kg}$ (appropriate for gestational age), cried immediately after birth, with APGAR scores of 8/10, 9/10. No obvious congenital anomalies seen and abdomen examination done was soft, no distension, tenderness or erythema.

Passed meconium on table. X-ray of abdomen taken at 1st hour of life showed soft tissue shadow on right side, pushing bowel to left and pediatric surgery opinion obtained immediately. Baby was started on expressed breast milk, which the baby tolerated well. USG abdomen was taken, showed large well defined thin intraperitoneal cystic lesion of size $5.7 \times 3.9 \times 6.3 \mathrm{~cm}$ with floating internal echoes and echogenic debris with thin internal septations, suggestive of a complex ovarian cyst with hemorrhage, highly indicative of torsion. No evidence of any component or internal vascularity noted within.

Hence, with high suspicion of ovarian torsion, baby was taken up for diagnostic laparoscopy under general anesthesia on second day of life, which showed large right ovarian cyst with torsion with adhesions to the small bowel. Uterus and left ovary were normal. $75 \mathrm{ml}$ of hemorrhagic fluid aspirated, and procedure was converted to laparoscopic ovarian cystectomy. Tissue sample was sent for histopathological examination, turned out to be simple cyst with features of torsion.

Baby was kept nil per oral and continued on IV fluids. Minimal tube feeds started by 6 hours post op and the baby tolerated well.

IV fluids tapered and oral feeds started on POD-1. Baby was discharged on day 7 of life, POD-5. Baby maintained saturation, hemodynamically stable, on direct breast feeds and tolerated well. Per abdomen examination was soft, no distension or tenderness and passed stools.

\section{Investigations}

USG done antenatally at 38 weeks of gestation showed multi loculated cyst in the right side of the fetal abdomen of size $6.3 \times 5 \times 5.37 \mathrm{~cm}$, likely a right gonadal cyst with hemorrhage. Post-delivery, on day 1 of life, X-ray of abdomen taken showed soft tissue shadow on right side, pushing bowel to left.

After which, USG abdomen was taken, showed large well defined thin intraperitoneal cystic lesion of size $5.7 \times 3.9 \times 6.3 \mathrm{~cm}$ with floating internal echoes and echogenic debris with thin internal septations, suggestive of a complex cyst at the sub-hepatic region and right lumbar quadrant of abdomen. No evidence of any component or internal vascularity noted within.

Hence, baby was taken up for diagnostic laparoscopy under general anesthesia on second day of life, which showed large right ovarian cyst with torsion with adhesions to the small bowel. Uterus and left ovary were normal. $75 \mathrm{ml}$ of hemorrhagic fluid aspirated.

\section{Differential diagnosis}

At 38 weeks of gestation, when ultrasound taken, a multiloculated hemorrhagic cyst was seen, which can likely be mesenteric cyst or ovarian cyst. In any antenatal mother at third trimester, with a female fetus, having a cyst in the lumbar quadrant should evoke a suspicion of ovarian cyst, while a thick wall cyst with bowel dilatation, then mesenteric cyst suspected. Any suspected ovarian cyst with hemorrhage points towards ovarian torsion, hence was diagnosed to be a hemorrhagic ovarian cyst with suspicion of torsion.

\section{Treatment}

During pregnancy, patient was counselled regarding the cyst seen in the fetal abdomen. Post-delivery, after appropriate investigations, baby was taken up for diagnostic laparoscopy under general anesthesia on second day of life, which showed large right ovarian cyst with torsion with adhesions to the small bowel. Uterus and left ovary were normal. $75 \mathrm{ml}$ of hemorrhagic fluid aspirated, and procedure was converted to laparoscopic ovarian cystectomy. Cyst was removed and tissue sample was sent for histopathological examination, turned out to be simple cyst with features of torsion.

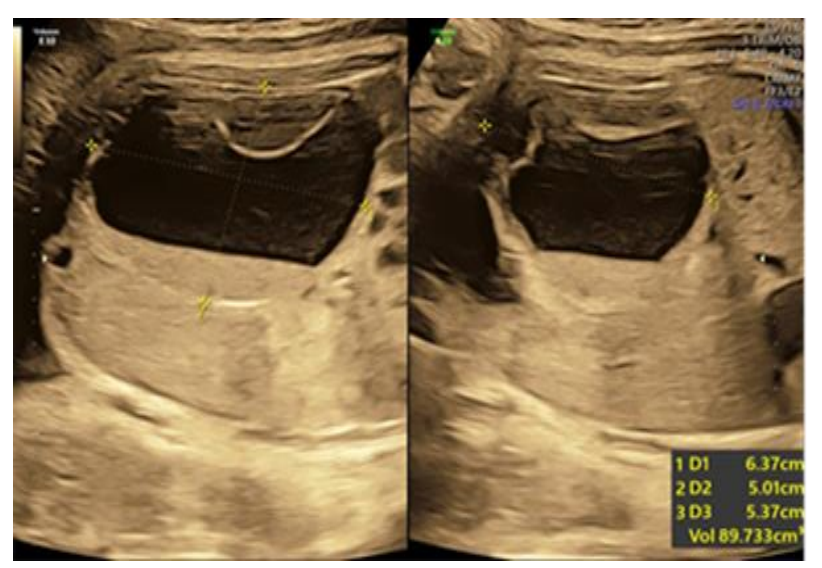

Figure 1: Growth scan. 


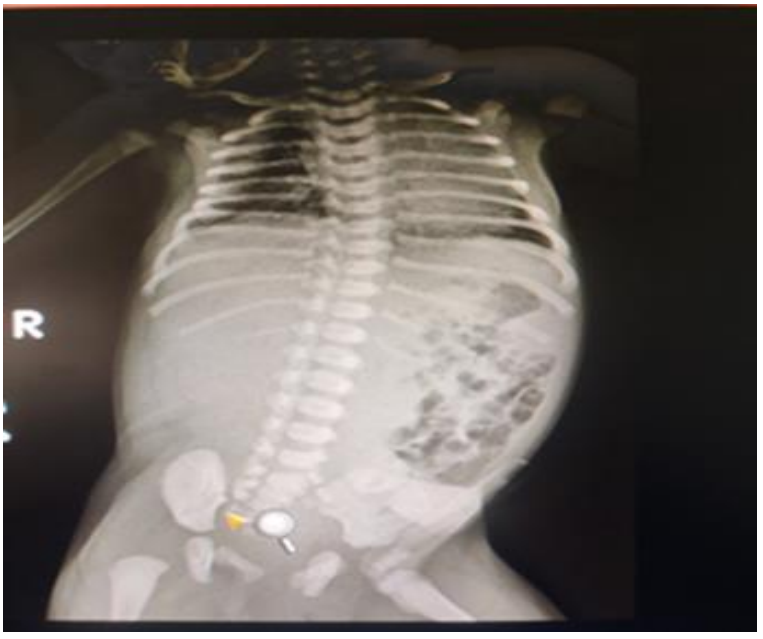

Figure 2: X-Ray of baby on day 1 of life.

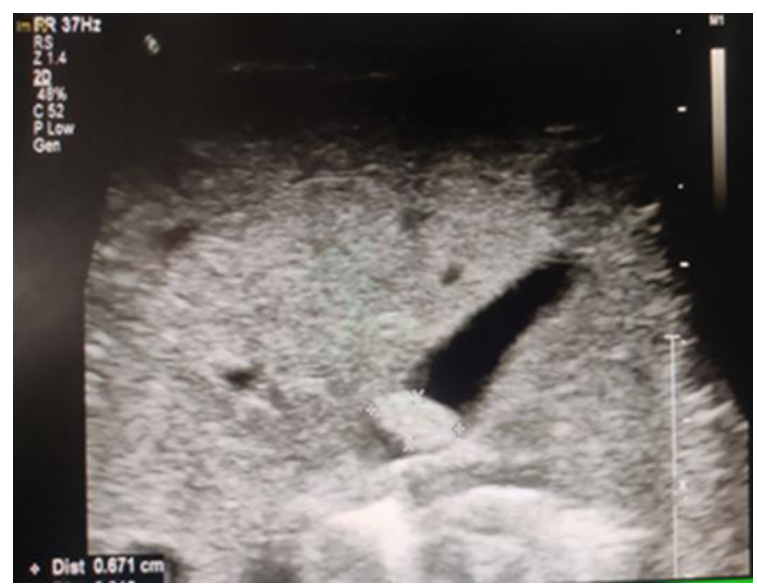

Figure 3: USG abdomen of baby.

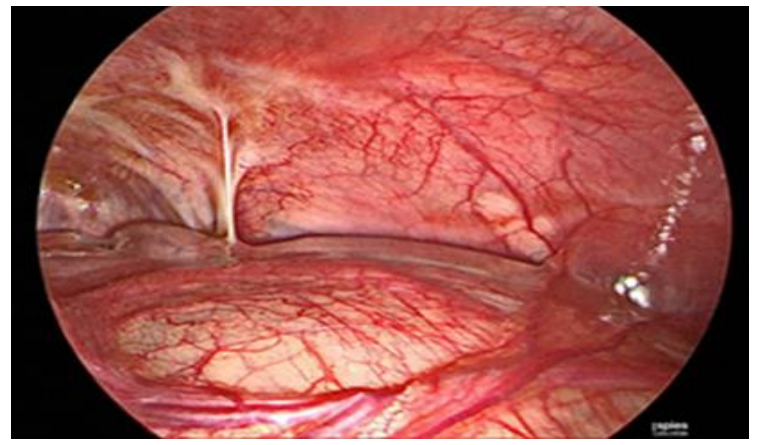

Figure 4: Diagnostic laparoscopy.

Baby was kept nil per oral and continued on IV fluids. Minimal tube feeds started by 6 hours post op and the baby tolerated well. IV fluids tapered and oral feeds started on POD-1. Baby was discharged on day 7 of life, POD-5.

Baby maintained saturation, hemodynamically stable, on direct breast feeds and tolerated well. Per abdomen examination was soft, no distension or tenderness and passed stools.

\section{Outcome and follow-up}

After laparoscopic cystectomy, baby was kept nil per oral and continued on IV fluids. Minimal tube feeds started by 6 hours post op and the baby tolerated well. IV fluids tapered and oral feeds started on POD-1.

Baby was discharged on day 7 of life, POD-5. Baby maintained saturation, hemodynamically stable, on direct breast feeds and tolerated well. Per abdomen examination was soft, no distension or tenderness and passed stools.

\section{DISCUSSION}

Fetal ovarian cysts are the most common abdominal masses in fetuses and neonates. The earliest age at which a fetal ovarian cyst detected is at $19^{\text {th }}$ week of gestation, but usually identified arounds 28 weeks gestation. ${ }^{1}$ Any antenatal mother at third trimester, with a female fetus, having a cyst in the lumbar quadrant should be suspected of ovarian cyst, while a thick wall cyst with bowel dilatation, then mesenteric cyst suspected. The most common complication of large ovarian cyst is torsion. Ovarian torsion results from partial or complete twist of ovary and fallopian tube. USG findings of ovarian torsion are variable echogenicity, ovarian edema, free pelvic fluid and absent blood flow on Doppler. Initially, there will be compromise of lymphatic drainage, followed by venous obstruction and hemorrhagic infarction, leading to gangrene, infection and peritonitis. For these in-utero fetal ovarian cysts, antenatal interventions are very rare, although cyst decompression can be done. Postnatally, all complex cysts and simple cysts of $>6 \mathrm{~cm}$ size need to be removed, while simple cysts $<6 \mathrm{~cm}$, regular check-ups and reassurance is sufficient. ${ }^{3}$

The importance of aspiration of ovarian cysts exceeding a $40 \mathrm{~mm}$ diameter was reported by Noia et al. The authors emphasised that aspiration of ovarian cysts allows a good longitudinal treatment of foetal affection, thus avoiding torsion, tissue necrosis and postnatal surgery. The suggested threshold size for prenatal aspiration of simple cysts varied from 30 to $50 \mathrm{~mm}$ in most reports. Complications of large cysts include the impairment of spontaneous delivery, distension of the fetal abdomen and disruption of the fetal heart function. Another study by Dimitraki et al suggested treatment of symptomatic cysts or cysts with a diameter of $>5 \mathrm{~cm}$ which do not regress or enlarge, whereas another report suggested neonatal surgery in the case of complex cysts regardless of size or in simple cysts larger than $20 \mathrm{~mm}$ in diameter. Surgical methods via laparoscopy or laparotomy can be performed depending on the experience of the surgeon. Two-port laparoscopy has been used in the management of fetal ovarian cyst that is cosmetically preferable and allows earlier feeding and recovery. Moreover, fetal ovarian carcinoma has been reported by Ziegler in a 30-weeks fetus. A review of the literature reveals that $85-90 \%$ of fetal ovarian masses are cystic (follicular or luteinic origins), and $10-15 \%$ are organic (3\% carcinomas and 7 - 
$12 \%$ represented by teratomas, and mucinous and serous cystadenomas). ${ }^{4}$

\section{CONCLUSION}

Fetal ovarian cysts, being the most common abdominal masses in fetuses and neonates, are detected earliest by 19 weeks of gestation, but usually identified arounds 28 weeks gestation. The most common complication of large ovarian cyst is torsion, which results from partial or complete twist of ovary and fallopian tube. USG findings of ovarian torsion are variable echogenicity, ovarian edema, free pelvic fluid and absent blood flow on Doppler. Due to impaired blood supply, can lead to gangrene, infection and peritonitis. For these in-utero fetal ovarian cysts, antenatal interventions are very rare, although cyst decompression can be done. Postnatally, all complex cysts and simple cysts of $>6 \mathrm{~cm}$ size need to be removed, while simple cysts $<6 \mathrm{~cm}$, regular check-ups and reassurance is sufficient.

Funding: No funding sources Conflict of interest: None declared

Ethical approval: Not required

\section{REFERENCES}

1. Sheth R, Hoelzer D, Scattergood E, Germaine P. In Utero Fetal Ovarian Torsion with Imaging Findings on Ultrasound and MRI. Case rep Rad. 2012;1-3.
2. Perrotin F, Potin J, Haddad G, Taveau C, Lansac J, Body G. Fetal ovarian cysts: a report of three cases managed by intrauterine aspiration. Ultrasound Obstet Gynecol. 2000;16(7):655-9.

3. Mathews MAB, Raval MB, Watkins DJ, King D. Diagnosis and management of an ovarian cyst complicated by torsion in utero- A case report. J Pediat Surg Case Rep. 2014;2(1):20-2.

4. Erol O, Erol MB, Isenlik BS, Ozkiraz S, Karaca M. Prenatal diagnosis of fetal ovarian cyst: case report and review of the literature. J Turk Ger Gynecol Assoc. 2013;14(2):119-22.

5. Singal AK, Vignesh KG, Paul S, Matthai J. Antenatally diagnosed ovarian cyst with torsion managed laparoscopically. J Indian Assoc Pediatr Surg. 2008;13(1):28-9.

6. Nussbaum AR, Sanders RC, Hartman DS, Dudgeon DL, Parmley TH. Neonatal ovarian cysts: sonographic-pathologic correlation. Radiology. 1988;168(3):817-21.

7. Mudholkar VG, Acharya AS, Kulkarni AM, Hirgude ST. Antenatally diagnosed neonatal ovarian cyst with torsion. Indian J Pathol Microbiol. 2011;54(1):228-9.

Cite this article as: Balasubramanian KK, Natarajan L, Arunachalam P, Maheshwari L. Torsion of inutero fetal ovarian cyst. Int J Reprod Contracept Obstet Gynecol 2022;11:965-8. 